\title{
Crenolanib, a PDGFR inhibitor, suppresses lung cancer cell proliferation and inhibits tumor growth in vivo
}

This article was published in the following Dove Press journal:

OncoTargets and Therapy

26 September 2014

Number of times this article has been viewed

\section{Ping Wang' \\ Liqiang Song ${ }^{2}$ \\ Hui $\mathrm{Ge}^{\prime}$ \\ Pule Jin' \\ Yifang Jiang' \\ Wenxia $\mathrm{Hu}^{\prime}$ \\ Nan Geng'}

'Department of Respiratory Medicine, Fourth Hospital of Hebei Medical University, Shijiazhuang, Hebei, People's Republic of China; ${ }^{2}$ Department of Pathology, School of Basic Medical Sciences, Peking Union Medical College, Beijing, People's

Republic of China
Correspondence: Ping Wang 12 jiankang Rd, Qiaodong, Shijiazhuang, Hebei 0500I I, People's Republic of China Tel +86 I393II30696

Email pingwang1393@gmail.com
Abstract: Platelet-derived growth factor (PDGF) and its receptors (PDGFR), including PDGFR $\alpha$ and PDGFR $\beta$, play important roles in tumorigenesis, tumor progression, and the regulation of stromal cell function. Constitutive activation of PDGFR signaling, gene rearrangement, and activating mutations of PDGFR have been identified in various types of human tumors and malignancies. PDGFR $\alpha$ and PDGFR $\beta$ belong to the family of type III receptor tyrosine kinases and, upon stimulation, activate downstream signaling cascades. Crenolanib is a specific tyrosine kinase inhibitor that targets and inhibits the kinase activity of PDGFR and the FMS-related tyrosine kinase 3. Its clinical efficacy in several human tumors is currently under investigation in Phase II clinical trials. In this study, we examined the potential role of crenolanib in the treatment of non-small-cell lung cancer (NSCLC). Using A549 cells as a model system, we have shown that crenolanib is capable of suppressing proliferation and inducing apoptosis in a dose-dependent manner. Crenolanib-treated cells have reduced migratory activity in response to inducers of chemotaxis. Furthermore, the in vivo antitumor activity of crenolanib was confirmed in an NSCLC xenograft tumor model. Injection of crenolanib significantly inhibited the growth of tumor mass by inducing apoptosis in tumor cells. Our results provide strong evidence supporting the use of crenolanib as a potential therapeutic agent in treating NSCLC. This work sets a foundation for further development of targeted and personalized therapeutics for lung cancer.

Keywords: platelet-derived growth factor receptor signaling, receptor tyrosine kinase, tyrosine kinase inhibitor, non-small-cell lung cancer, chemotherapy, targeted therapy

\section{Introduction}

Platelet-derived growth factor receptors (PDGFRs), including PDGFR $\alpha$ and PDGFR $\beta$, belong to the family of cell surface type III receptor tyrosine kinases (RTKs). ${ }^{1,2}$ Upon binding of the ligands, platelet-derived growth factors (PDGFs), the receptor complex is activated and the cytosolic domains serve as docking sites for coactivators and subsequently initiate downstream signaling cascades such as MAPK, PI3K, and STAT3 pathways. ${ }^{3-6}$ PDGFR signaling regulates a variety of biological processes, including cellular growth, cellular differentiation, cell migration, and angiogenesis. ${ }^{1,7}$ Deregulated PDGFR signaling has been implicated in the pathogenesis of several human diseases and malignancies. ${ }^{8-11}$ For example, in patients with gastrointestinal stromal tumors, chronic myelomonocytic leukemia, and glioblastoma multiforme, mutations have been identified in the genes encoding PDGFR, which results in constitutive activation of the kinase activity, overstimulation of signal transduction, interaction with adjacent stroma and vasculature, and eventually autocrine regulation of tumor cell growth. ${ }^{12-15}$ Therefore, the 
PDGFR pathway represents a potential therapeutic target in patients harboring activating mutations of PDGFR.

With PDGFR as a promising target in cancer therapeutics, efforts have been made to develop specific kinase inhibitors that disrupt the receptor activation of PDGFR. Crenolanib is a specific tyrosine kinase inhibitor that targets and inhibits the kinase activity of PDGFR and the FMS-related tyrosine kinase 3 (FLT3). ${ }^{16-18}$ In a completed Phase I clinical trial, its pharmacokinetics and safety were evaluated and the recommended dosage was well tolerated by patients. ${ }^{16}$ The clinical efficacy of crenolanib in several human tumors is currently under investigation in multiple Phase II clinical trials. Unlike other RTK inhibitors such as imatinib mesylate and motesanib, which have been previously studied and tested in clinical trials for various types of cancer, crenolanib preferentially targets the phosphorylated, thus active, form of PDGFR that is often derived from constitutively active mutations of genes encoding PDGFR. ${ }^{17,19,20}$ Therefore, crenolanib may provide targeted therapy to patients with PDGFR mutations and have reduced toxicity compared with other broad-spectrum multikinase inhibitors.

In this study, we aim to test the potential efficacy of crenolanib in treating non-small-cell lung cancer (NSCLC), a collective type of epithelial lung carcinoma. Previous research has suggested a strong correlation between deregulated PDGFR signaling and NSCLC tumor progression. ${ }^{21-24}$ Expression and activity of PDGF and PDGFR have been used as prognostic indicators in lung carcinoma. The traditional treatment for NSCLCs involves surgical resection followed by applications of antiangiogenic agents such as targeted cytotoxic drugs. ${ }^{25,26}$ A549 cells were derived from human lung carcinoma and express PDGFR $\alpha$ in high levels. ${ }^{27} \mathrm{By}$ inhibiting the PDGFR signaling, our data demonstrated that crenolanib treatment suppressed the proliferation and migration of A549 cells and inhibited tumor growth in an NSCLC xenograft tumor model. Our study provides strong evidence supporting the use of crenolanib as a personalized and effective second-line therapy for NSCLC patients.

\section{Materials and methods}

\section{Reagents, cells, and mice}

Crenolanib (CP-868596) was purchased from Selleck Chemicals LLC (Houston, TX, USA). Human NSCLC cell line A549 was purchased from the American Type Culture Collection (Rockville, MD, USA). A549 cells were maintained in RPMI medium supplemented with $10 \%$ heatinactivated fetal bovine serum, $2 \mathrm{mM} \mathrm{L-glutamine,} \mathrm{and} 100 \mathrm{U} /$ $\mathrm{mL}$ penicillin-streptomycin in a humidified incubator at $37^{\circ} \mathrm{C}$ in $5 \% \mathrm{CO}_{2}$. The athymic nude mice were purchased from Slack Company (Shanghai, People's Republic of China). Care and treatment of mice were in accordance with the Animal Care and Use Committee guidelines of Hebei Medical University (Shijiazhuang, Hebei, People's Republic of China).

\section{Cell viability assay}

Cells were seeded in 96-well plates in triplicate and treated with different doses of crenolanib for 24,48 , or 72 hours as indicated in Figure 1. Cell viability at the end of each treatment was measured using CellTiter-Glo Assay Kit (Promega, Madison, WI, USA) according to the manufacturer's instructions. Briefly, an equal volume of the CellTiter-Glo reagent $(100 \mu \mathrm{L})$ was added directly to the cell culture in 96-well plates and the plates were placed on an orbital shaker for 2 minutes followed by incubation at room temperature for 10 minutes. Luminescence was read in a luminometer (Molecular Devices, Sunnyvale, CA, USA).

\section{Flow cytomery analysis}

Cells treated with different concentrations of crenolanib were harvested, fixed, and stained with propidium iodide using a BD Cycletest Plus DNA Reagent Kit (Becton Dickinson, Franklin lakes, NJ, USA). Cells were collected and analyzed for deoxyribonucleic acid (DNA) content using FACSCalibur (BD Biosciences, San Jose, CA, USA) and CellQuest Pro flow cytometry analytic software (Becton Dickinson).

\section{Nuclei staining}

Cells were seeded on polylysine-coated glass coverslips and treated with different concentrations of crenolanib as indicated in Figure 2. After 48 hours, cells were fixed with 4\% paraformaldehyde for 10 minutes and stained with Hoechst (SigmaAldrich, St Louis, MO, USA) for 10 minutes. Images were obtained and analyzed using an Olympus BX51 fluorescent microscope (Olympus America Inc., Melville, NY, USA).

\section{Transwell assay}

$1 \times 10^{5}$ cells in RPMI media supplemented with $1 \%$ fetal bovine serum were seeded into the upper compartments of the 24-transwell Boyden chamber (Costar, Bedford, MA, USA). Different concentrations of crenolanib were added to cells as indicated in Figure 3. Medium $(650 \mu \mathrm{L})$ supplemented with $10 \%$ fetal bovine serum was loaded into the lower chambers to be used as a chemoattractant. After incubation for 12 hours, the nonmigrating cells were wiped off from the upper surface, and the migrated cells on the lower side were fixed, stained with $0.1 \%$ crystal violet, and photographed using an Eclipse LV150L microscope (Nikon Corporation, Tokyo, Japan). For absorbance measurement, the migrated cells on the lower side of the chamber were lysed with $10 \%$ acetic acid and measured at $595 \mathrm{~nm}$. 


\section{Efficacy studies in a mouse xenograft model in vivo}

A549 cells were injected into the axillary regions of mice $\left(2 \times 10^{6}\right.$ cells/mouse $)$. When the tumor volumes reached $70 \mathrm{~mm}^{3}$, the mice were randomly allocated to the control group, low-dose crenolanib group $(10 \mathrm{mg} / \mathrm{kg})$, or highdose crenolanib group $(20 \mathrm{mg} / \mathrm{kg})(\mathrm{n}=6$ per group). The vehicle for crenolanib treatment consists of $10 \% 1$-methyl2-pyrrolidinone and 90\% polyethylene glycol 300 (SigmaAldrich). The tumor size and mouse body weight were measured every other day for about 2 weeks. The tumor volume was calculated as follows: $\left(\mathrm{mm}^{3}\right)=($ width $\times$ width $\times$ length $/ 2$. After treatment, the mice were euthanized using carbon dioxide, and the tumors were harvested and analyzed.

\section{Immunohistochemistry analysis}

Tumor samples were harvested from mice, trimmed into $5 \mathrm{~mm}^{3}$ pieces, and immediately fixed in $10 \%$ neutral buffered formalin. After fixation, the samples were transferred to $70 \%$ ethanol, embedded into paraffin blocks, and sectioned in $5 \mathrm{~mm}$ thicknesses. Terminal deoxynucleotidyl transferase dUTP nick end labeling (TUNEL) assay was performed using FragEL kit (Calbiochem, Darmstadt, Germany) according to the manufacturer's instructions. Briefly, slides were stained with TUNEL reagent for 1 hour followed by incubation with an Alexa Fluor 488-conjugated antibody for 30 minutes. The nuclei were stained with Hoechst for 20 minutes. The slides were photographed using an Olympus BX51 fluorescent microscope (Olympus America Inc.).

\section{Statistics}

Student's $t$-test and analysis of variance were performed using StatView (SAS Institute, Cary, NC, USA). The data shown are the mean values of triplicate measurements with error bars corresponding to standard deviation. $P<0.05$ was considered statistically significant.

\section{Results}

\section{Crenolanib decreases NSCLC cell viability}

PDGFR signaling promotes cell survival through activation of various downstream signal transduction cascades, including MAPK, PI3K, and STAT3 pathways. To determine whether inhibition of PDGFR signaling affects cellular proliferation, the A549 NSCLC cells were treated with crenolanib with a series of concentrations for 72 hours, and the cell viability was measured. We observed a dose-dependent inhibition of cancer cell viability (Figure 1A). To find out the onset of action for crenolanib, A549 cells were treated with crenolanib (50 $\mathrm{nM}$ ) for 24,48 , or 72 hours, followed by measurement of cell viability. The inhibitory effect of crenolanib was observed as early as 24 hours after treatment (Figure 1B).

\section{Crenolanib induces apoptosis in NSCLC cells}

PDGF is a potent mitogenic factor that enhances proliferation and antagonizes apoptosis. We further tested whether treatment of crenolanib was capable of inducing apoptosis in cancer cells. After treatment with crenolanib for 48 hours, the sub-G1 DNA content of A549 cells was measured as an indicator of late stage apoptosis. ${ }^{28}$ Similar to the previous observation in cell viability, crenolanib caused increased sub-G1 DNA content in a dosedependent manner, indicating that inhibition of PDGFR signaling promoted cells to undergo apoptosis (Figure 2A). Apoptosis-induced condensed and fragmented DNA is visualized in Figure 2B. Consistently, the number of cells

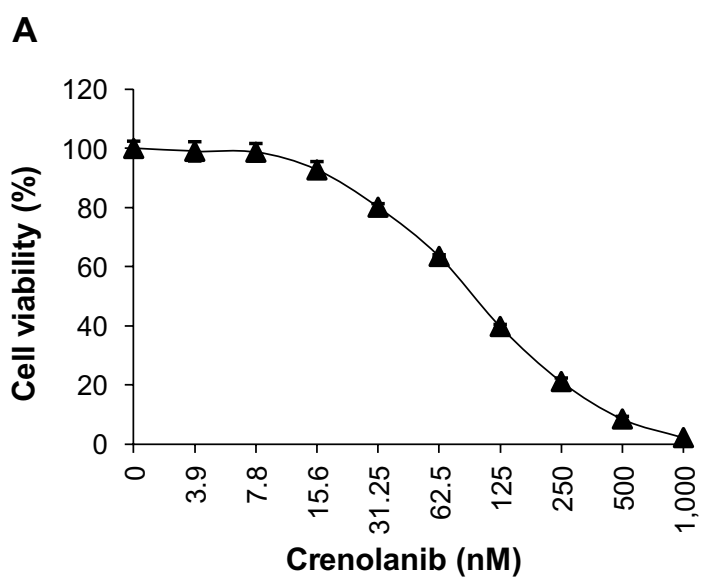

B

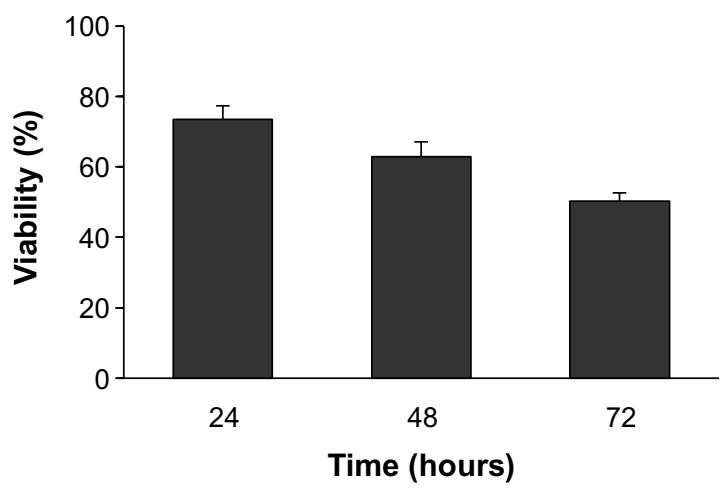

Figure I Crenolanib decreases non-small-cell lung cancer cell viability. Notes: (A) A549 cells were treated with crenolanib (3.9 1,000 nM) for 72 hours followed by measurement with cell viability assay. (B) A549 cells were treated with crenolanib $(50 \mathrm{nM})$ for 24,48 , or 72 hours followed by measurement with cell viability assay. In both experiments, viability of cells without crenolanib treatment was set to $100 \%$. 


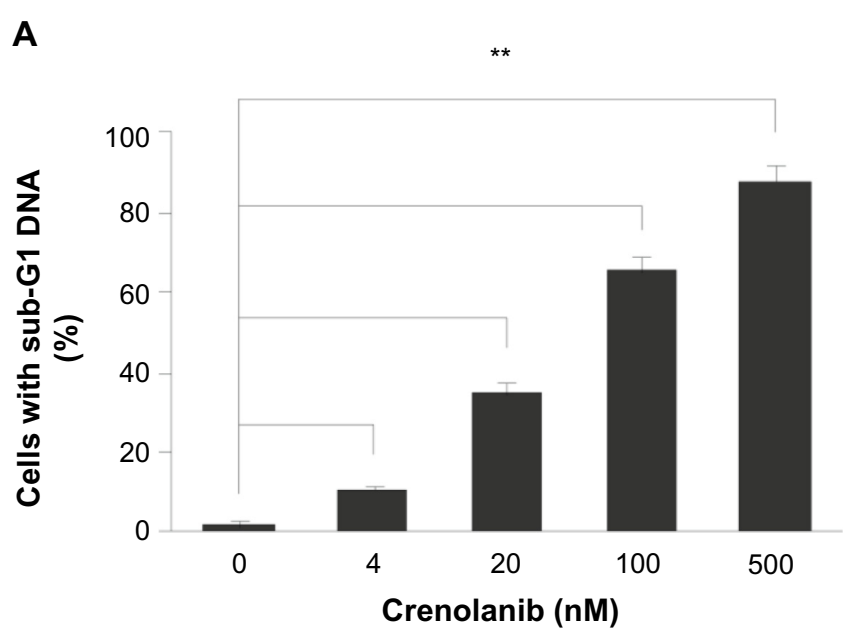

B

Control

Crenolanib $(500 \mathrm{nM})$
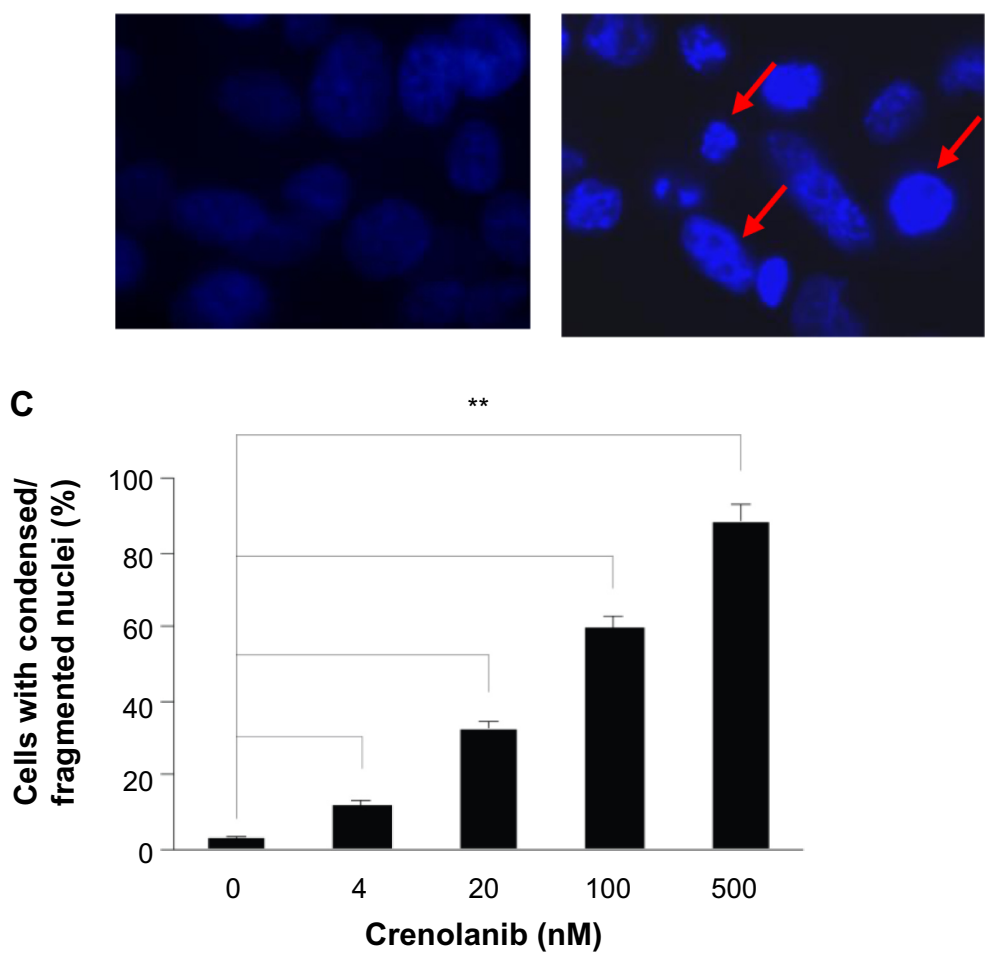

Figure 2 Crenolanib induces apoptosis in non-small-cell lung cancer cells.

Notes: (A) A549 cells were treated with crenolanib at the indicated concentrations for 48 hours followed by propidium iodide staining and flow cytometry analysis for sub-GI deoxyribonucleic acid (DNA) content. (B) A549 cells were incubated with crenolanib (500 nM) for 48 hours. The nuclei were stained with Hoechst and analyzed using a fluorescent microscope. The representative images are shown. Red arrows indicate apoptotic cells with condensed or fragmented DNA. (C) The number of cells with condensed/fragmented nuclei was quantitated by counting in five random fields. Percentage was calculated and the averaged results are shown.

Note: ${ }^{* *} P<0.01$.

with condensed/fragmented DNA increased proportionally with crenolanib treatment (Figure 2C).

\section{Crenolanib inhibits cell migration in NSCLC cells}

PDGF is a critical chemoattractant that binds to its cell surface receptor and regulates cell proliferation and chemotaxis. ${ }^{29}$
Malignant tumor cells are capable of migrating and invading to a second site for tumorigenesis. PDGFR signaling plays an important role in this process and the following angiogenesis to contribute to the metastasis of tumors. Therefore, PDGF/ PDGFR becomes an attractive target for cancer therapeutics.

We next investigated the effect of PDGFR inhibition on cancer cell migration. As shown in Figure 3A and B, treatment 
A

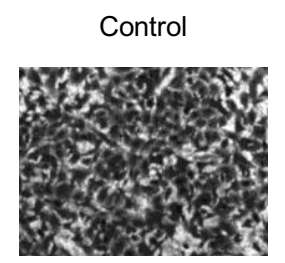

Crenolanib (12.5 nM)

Crenolanib $(25 \mathrm{nM})$

Crenolanib (50 nM)
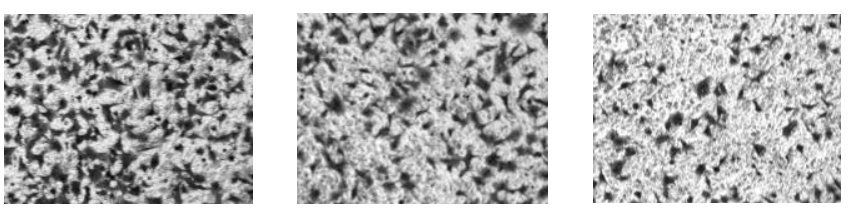

B

**

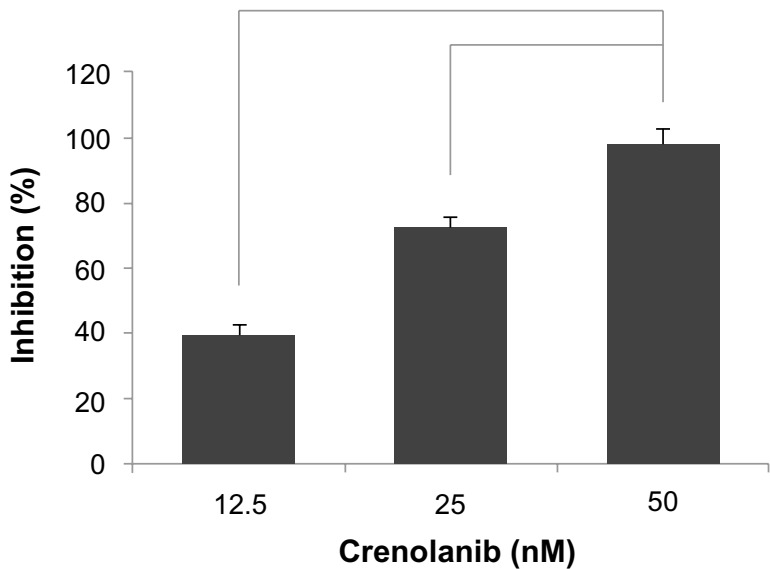

Figure 3 Crenolanib inhibits cell migration in non-small-cell lung cancer cells.

Notes: (A) A549 cells were treated with crenolanib (12.5, 25, or $50 \mathrm{nM}$ ) for 10 hours. The nonmigrated cells on the upper chamber of the transwell were removed and the migrated cells on the lower side were stained and photographed. The representative images are shown. (B) The migrated cells were lysed and colorimetric determination was made at $595 \mathrm{~nm}$. Summary of the quantitation of the inhibition from transwell assay is shown.

Note: $* * P<0.01$

with crenolanib inhibited the migration of A549 cells in a dose-dependent manner. This inhibition is unlikely due to the cytotoxicity of crenolanib, as the duration of treatment was significantly shorter and the concentrations were significantly lower compared with the conditions used in Figure 1.

\section{Crenolanib suppresses NSCLC tumor growth in vivo}

We then went on to investigate the antitumor effect of crenolanib in vivo. A549 cells were injected into the axillary regions of athymic nude mice. After successful inoculation, crenolanib $(10 \mathrm{mg} / \mathrm{kg}$ and $20 \mathrm{mg} / \mathrm{kg})$ was administered, and the tumor volumes were measured every other day. Crenolanib significantly inhibited the growth of tumor mass, and the strongest inhibitory effect was observed with $20 \mathrm{mg} / \mathrm{kg}$ treatment (Figure 4A). Consistent with the in vitro studies, crenolanib induced massive apoptosis in tumor cells, which was demonstrated by significantly increased DNA fragmentation detected in both TUNEL assay and nuclei staining (Figure 4B). Furthermore, the dosage of crenolanib applied was well tolerated by recipient mice. No weight loss was observed during the course of treatment (Figure 4C). Taken together, these results indicate that crenolanib can effectively suppress tumor growth in vivo by inducing apoptosis of tumor cells.

\section{Discussion}

Activating mutation in genes encoding critical signaling molecules has been identified in patients with different types of tumors, including NSCLC. For example, gene rearrangements and point mutations in the gene encoding the epidermal growth factor receptor have been found in a subset of NSCLC patients, and patients with such mutations had significantly increased response to treatment of gefitinib, a specific RTK inhibitor targeting the epidermal growth factor receptor. ${ }^{30-33}$ In another study, mutations in PDGFA, the gene encoding for PDGF $\alpha$, were identified in $13 \%$ of NSCLC patient tumor samples. ${ }^{34}$ Therefore, by selecting the RTK inhibitors with specific targeting capacity, effective chemotherapy can be directed to a targeted population.

Aberrant PDGFR signaling due to overexpression of the ligand PDGF or activating mutations of PDGFR has been suggested to be a critical player in tumorigenesis and angiogenesis of a variety of tumors. Blocking the PDGFR signaling by either specific neutralizing antibodies that target the receptors or RTK inhibitors that disrupt the kinase activity 

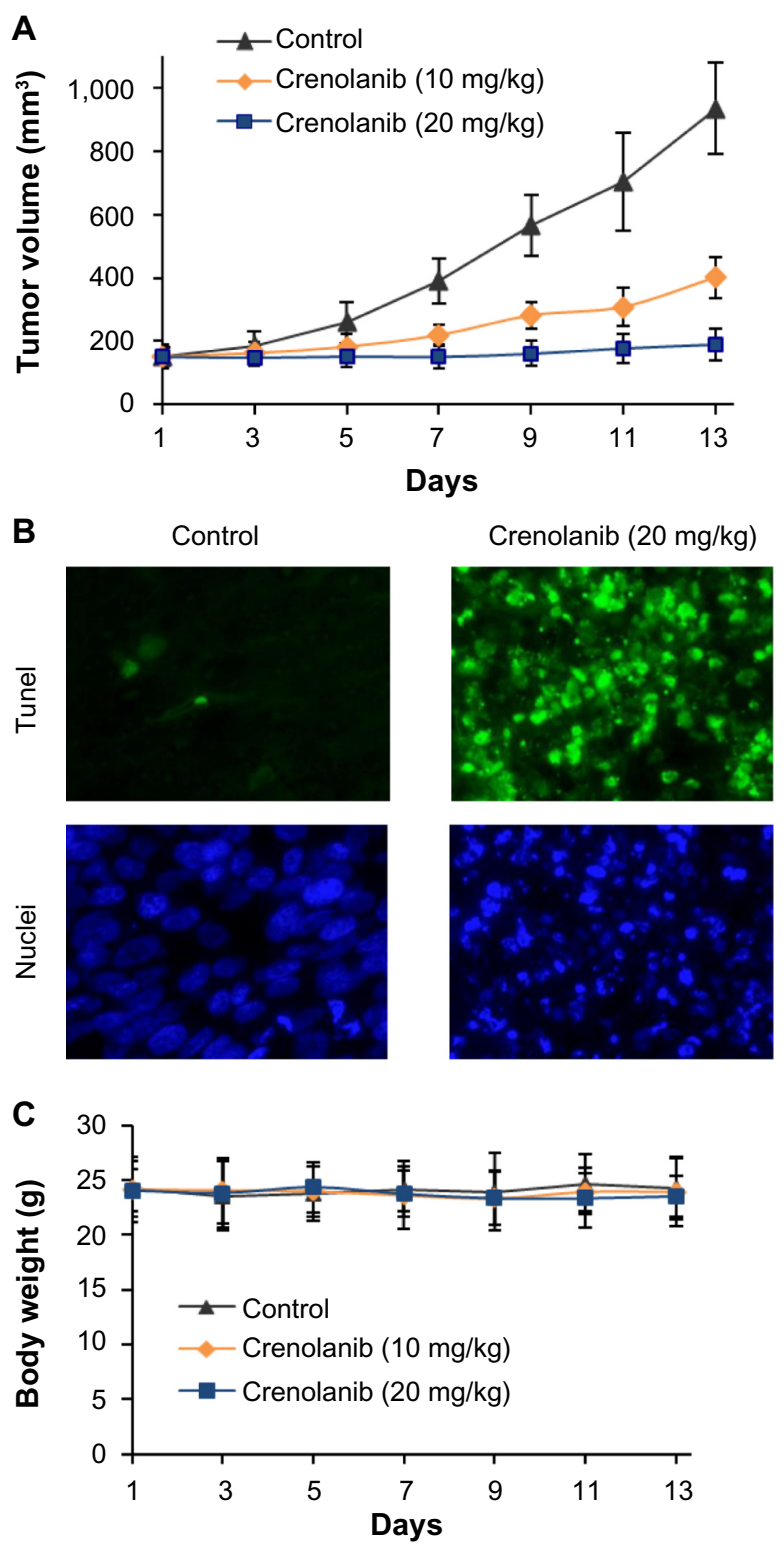

Figure 4 Crenolanib suppresses non-small-cell lung cancer tumor growth in vivo and induces tumor cell apoptosis.

Notes: (A) After inoculation of A549 cells, crenolanib (10 mg/kg and $20 \mathrm{mg} / \mathrm{kg}$ ) was injected into the nude mice. The tumors were measured every other day for about 2 weeks and the tumor volumes are shown. (B) After treatment of crenolanib for 2 weeks, tumors were harvested. Crenolanib induced apoptosis of tumor cells measured with terminal deoxynucleotidyl transferase dUTP nick end labeling assay (green) and nuclei staining with Hoechst (blue). (C) Body weight of mice during the course of treatment was measured every other day. Crenolanib had no significant cytotoxic effects on the body weight of mice during the treatments.

has proven to have clinical efficacy in treating a number of human malignancies, including NSCLC. ${ }^{35-38}$ Criteria for selecting candidate RTK inhibitors in cancer therapy include minimal toxicity and higher affinity with mutated forms of the kinase targets that are frequently present in cancer patients. Crenolanib, a potent RTK inhibitor with narrow and selective reactivity against both wild-type and mutated FLT3 and PDGFR (including PDGFR $\alpha$ and PDGFR $\beta$ ), represents the first anticancer RTK inhibitor with predictably minimal toxicity. Previous research and clinical trials have validated the therapeutic efficacy of crenolanib in gastrointestinal stromal tumors and human acute myeloid leukemia. ${ }^{16,18-20}$ Our results provide additional evidence to demonstrate that crenolanib can yield clinical benefit to NSCLC patients with deregulated PDGFR signaling.

In this study, we found that treatment with crenolanib inhibited growth and induced apoptosis of NSCLC cells in a dose- and time-dependent manner, which was consistent with a previous study in other tumor cell lines. ${ }^{17}$ Treating tumor cells with crenolanib resulted in decreased phosphorylation and thus reduced activity of downstream signaling molecules, including AKT3, MAPK, and STAT5, ${ }^{17}$ which we believe led to inhibition of cell proliferation. However, the mechanism of crenolanib-induced apoptosis has not been elucidated. It will be informative to examine the status of apoptosis marker molecules before and after crenolanib treatment. Caspase-3 cleavage, ratio of anti- and proapoptotic proteins, level of HSP90 client proteins, and other apoptosisrelated pathways should be thoroughly assessed. In addition, crenolanib-treated NSCLC cells displayed reduced migratory activity in response to inducers of chemotaxis, which implies impaired angiogenesis. Although this hypothesis is tempting, solid data from in vitro and/or in vivo angiogenesis assays are required. Furthermore, injection of crenolanib significantly inhibited the growth of NSCLC tumor cells in vivo. This is the first report that documents the antitumor activity of crenolanib in NSCLC cells. This work could set a foundation for further development of targeted and personalized therapeutics for lung cancer.

The role of PDGFR signaling in interaction between tumor cells and stroma has also been suggested by previous studies. ${ }^{11,39,40}$ Stromal fibroblasts are recruited to the site of the tumor and contribute to the growth of tumor mass and the angiogenic process. In a mouse xenograft model of lung carcinoma, highest expression and activity of PDGFR $\alpha$ have been detected in the tumor-surrounding stroma, and when the PDGFR signaling is blocked by neutralizing soluble recombinant PDGFR, tumor growth and tumor invasion are significantly inhibited. ${ }^{11}$ These findings indicate that suppressing PDGFR signaling has an antitumor effect on both tumor cells and the microenvironment. Further investigation can be performed to assess the effect of crenolanib in tumor stroma and fibroblast recruitment. If crenolanib could affect both tumor survival and the angiogenic process, it may lead to great clinical benefit in patients with advanced or metastasized NSCLC. 


\section{Disclosure}

The authors declare no conflicts of interest in this work.

\section{References}

1. Heldin $\mathrm{CH}$. Structural and functional studies on platelet-derived growth factor. EMBO J. 1992;11(12):4251-4259.

2. Shim AH, Liu H, Focia PJ, Chen X, Lin PC, He X. Structures of a platelet-derived growth factor/propeptide complex and a plateletderived growth factor/receptor complex. Proc Natl Acad Sci U S A. 2010;107(25):11307-11312

3. Williams LT. Signal transduction by the platelet-derived growth factor receptor. Science. 1989;243(4898):1564-1570.

4. Valius M, Kazlauskas A. Phospholipase C-gamma 1 and phosphatidylinositol 3 kinase are the downstream mediators of the PDGF receptor's mitogenic signal. Cell. 1993;73(2):321-334.

5. Montmayeur JP, Valius M, Vandenheede J, Kazlauskas A. The plateletderived growth factor beta receptor triggers multiple cytoplasmic signaling cascades that arrive at the nucleus as distinguishable inputs. J Biol Chem. 1997;272(51):32670-32678.

6. Blazevic T, Schwaiberger AV, Schreiner CE, et al. 12/15-lipoxygenase contributes to platelet-derived growth factor-induced activation of signal transducer and activator of transcription 3. J Biol Chem. 2013;288(49):35592-35603.

7. Hoch RV, Soriano P. Roles of PDGF in animal development. Development. 2003;130(20):4769-4784.

8. Coltrera MD, Wang J, Porter PL, Gown AM. Expression of plateletderived growth factor B-chain and the platelet-derived growth factor receptor beta subunit in human breast tissue and breast carcinoma. Cancer Res. 1995;55(12):2703-2708.

9. MacDonald TJ, Brown KM, LaFleur B, et al. Expression profiling of medulloblastoma: PDGFRA and the RAS/MAPK pathway as therapeutic targets for metastatic disease. Nat Genet. 2001;29(2):143-152.

10. Lokker NA, Sullivan CM, Hollenbach SJ, Israel MA, Giese NA. Platelet-derived growth factor (PDGF) autocrine signaling regulates survival and mitogenic pathways in glioblastoma cells: evidence that the novel PDGF-C and PDGF-D ligands may play a role in the development of brain tumors. Cancer Res. 2002;62(13):3729-3735.

11. Tejada ML, Yu L, Dong J, et al. Tumor-driven paracrine platelet-derived growth factor receptor alpha signaling is a key determinant of stromal cell recruitment in a model of human lung carcinoma. Clin Cancer Res. 2006;12(9):2676-2688.

12. Heinrich MC, Corless CL, Duensing A, et al. PDGFRA activating mutations in gastrointestinal stromal tumors. Science. 2003;299(5607):708-710.

13. Corless CL, Schroeder A, Griffith D, et al. PDGFRA mutations in gastrointestinal stromal tumors: frequency, spectrum and in vitro sensitivity to imatinib. J Clin Oncol. 2005;23(23):5357-5364.

14. Hiwatari M, Ono R, Taki T, et al. Novel gain-of-function mutation in the extracellular domain of the PDGFRA gene in infant acute lymphoblastic leukemia with $\mathrm{t}(4 ; 11)(\mathrm{q} 21 ; \mathrm{q} 23)$. Leukemia. 2008;22(12):2279-2280.

15. Ozawa T, Brennan CW, Wang L, et al. PDGFRA gene rearrangements are frequent genetic events in PDGFRA-amplified glioblastomas. Genes Dev. 2010;24(19):2205-2218.

16. Lewis NL, Lewis LD, Eder JP, et al. Phase I study of the safety, tolerability, and pharmacokinetics of oral CP-868,596, a highly specific plateletderived growth factor receptor tyrosine kinase inhibitor in patients with advanced cancers. J Clin Oncol. 2009;27(31):5262-5269.

17. Galanis A, Ma H, Rajkhowa T, et al. Crenolanib is a potent inhibitor of FLT3 with activity against resistance-conferring point mutants. Blood. 2014;123(1):94-100.

18. Smith CC, Lasater EA, Lin KC, et al. Crenolanib is a selective type I panFLT3 inhibitor. Proc Natl Acad Sci U S A. 2014;111(14):5319-5324.

19. Heinrich MC, Griffith D, McKinley A, et al. Crenolanib inhibits the drug-resistant PDGFRA D842V mutation associated with imatinib-resistant gastrointestinal stromal tumors. Clin Cancer Res. 2012;18(16):4375-4384.
20. Zimmerman EI, Turner DC, Buaboonnam J, et al. Crenolanib is active against models of drug-resistant FLT3-ITD-positive acute myeloid leukemia. Blood. 2013;122(22):3607-3615.

21. Kawai T, Hiroi S, Torikata C. Expression in lung carcinomas of platelet-derived growth factor and its receptors. Lab Invest. 1997;77(5):431-436.

22. Negri T, Casieri P, Miselli F, et al. Evidence for PDGFRA, PDGFRB and KIT deregulation in an NSCLC patient. Br J Cancer. 2007;96(1):180-181.

23. Donnem T, Al-Saad S, Al-Shibli K, Andersen S, Busund LT, Bremnes RM. Prognostic impact of platelet-derived growth factors in non-small cell lung cancer tumor and stromal cells. J Thorac Oncol. 2008;3(9):963-970.

24. Kono SA, Heasley LE, Doebele RC, Camidge DR. Adding to the mix: fibroblast growth factor and platelet-derived growth factor receptor pathways as targets in non-small cell lung cancer. Curr Cancer Drug Targets. 2012;12(2):107-123.

25. Bauman JE, Eaton KD, Martins RG. Antagonism of platelet-derived growth factor receptor in non small cell lung cancer: rationale and investigations. Clin Cancer Res. 2007;13(15 Pt 2):S4632-S4636.

26. Cascone T, Gridelli C, Ciardiello F. Combined targeted therapies in non-small cell lung cancer: a winner strategy? Curr Opin Oncol. 2007;19(2):98-102.

27. Zhang P, Gao WY, Turner S, Ducatman BS. Gleevec (STI-571) inhibits lung cancer cell growth (A549) and potentiates the cisplatin effect in vitro. Mol Cancer. 2003;2:1.

28. Nicoletti I, Migliorati G, Pagliacci MC, Grignani F, Riccardi C. A rapid and simple method for measuring thymocyte apoptosis by propidium iodide staining and flow cytometry. $J$ Immunol Methods. 1991;139(2):271-279.

29. Kundra V, Escobedo JA, Kazlauskas A, et al. Regulation of chemotaxis by the platelet-derived growth factor receptor-beta. Nature. 1994;367(6462):474-476.

30. Hirsch FR, Herbst RS, Olsen C, et al. Increased EGFR gene copy number detected by fluorescent in situ hybridization predicts outcome in non-small-cell lung cancer patients treated with cetuximab and chemotherapy. J Clin Oncol. 2008;26(20):3351-3357.

31. Sequist LV, Martins RG, Spigel D, et al. First-line gefitinib in patients with advanced non-small-cell lung cancer harboring somatic EGFR mutations. J Clin Oncol. 2008;26(15):2442-2449.

32. Yang CH, Yu CJ, Shih JY, et al. Specific EGFR mutations predict treatment outcome of stage IIIB/IV patients with chemotherapy-naive non-small-cell lung cancer receiving first-line gefitinib monotherapy. J Clin Oncol. 2008;26(16):2745-2753.

33. Pallis AG, Syrigos KN. Epidermal growth factor receptor tyrosine kinase inhibitors in the treatment of NSCLC. Lung Cancer. 2013;80(2):120-130.

34. Rikova K, Guo A, Zeng Q, et al. Global survey of phosphotyrosine signaling identifies oncogenic kinases in lung cancer. Cell. 2007;131(6):1190-1203.

35. George D. Targeting PDGF receptors in cancer: rationales and proof of concept clinical trials. Adv Exp Med Biol. 2003;532:141-151.

36. Belani CP, Goss G, Blumenschein G Jr. Recent clinical developments and rationale for combining targeted agents in non-small cell lung cancer (NSCLC). Cancer Treat Rev. 2012;38(3):173-184.

37. Morth C, Valachis A. Single-agent versus combination chemotherapy as first-line treatment for patients with advanced non-small cell lung cancer and performance status 2: a literature-based meta-analysis of randomized studies. Lung Cancer. 2014;4(3):209-214.

38. Rashdan S, Hanna N. Nintedanib for the treatment of non-small-cell lung cancer. Expert Opin Pharmacother. 2014;15(5):729-739.

39. Dong J, Grunstein J, Tejada M, et al. VEGF-null cells require PDGFR alpha signaling-mediated stromal fibroblast recruitment for tumorigenesis. EMBO J. 2004;23(14):2800-2810.

40. Gerber DE, Gupta P, Dellinger MT, et al. Stromal platelet-derived growth factor receptor alpha (PDGFRalpha) provides a therapeutic target independent of tumor cell PDGFRalpha expression in lung cancer xenografts. Mol Cancer Ther. 2012;11(11):2473-2482. 


\section{Publish your work in this journal}

OncoTargets and Therapy is an international, peer-reviewed, open access journal focusing on the pathological basis of all cancers, potential targets for therapy and treatment protocols employed to improve the management of cancer patients. The journal also focuses on the impact of management programs and new therapeutic agents and protocols on

patient perspectives such as quality of life, adherence and satisfaction. The manuscript management system is completely online and includes a very quick and fair peer-review system, which is all easy to use. Visit http://www.dovepress.com/testimonials.php to read real quotes from published authors.

Submit your manuscript here: http://www.dovepress.com/oncotargets-and-therapy-journal 